\title{
Importancia de los Cambios Iniciales en la Psicoterapia con Adolescentes
}

\author{
Relevance of early changes in psychotherapy with adolescents
}

\author{
Olga Fernández \\ Universidad de Chile, Pontificia Universidad Católica de Chile \\ Carola Pérez \\ Universidad del Desarrollo, Pontificia Universidad Católica de Chile \\ Sergio Gloger \\ Psicomedica Research Group \\ Mariane Krause \\ Pontificia Universidad Católica de Chile
}

(Rec: 26 agosto 2015 / Acept: 17 octubre 2015)

\begin{abstract}
Resumen
Esta investigación tiene por objetivo estudiar los Indicadores de Cambio Genéricos (ICG) en psicoterapias con adolescentes, realizadas en contexto natural. Específicamente focaliza en la evolución de los ICG al interior de la etapa inicial de la terapia y su relación con la adherencia al tratamiento. Participaron 19 adolescentes, con diferentes diagnósticos, en modalidad de terapia individual de diferentes orientaciones teóricas. Evaluadores entrenados identificaron los ICG en las tres sesiones iniciales. Los resultados indican que se observa una evolución al interior de la etapa inicial de la terapia, en el sentido de un predominio de los indicadores jerárquicamente más bajos en la primera sesión en relación a la segunda y la tercera. Además los ICG máximos alcanzados en la fase inicial permiten predecir en forma significativa la probabilidad de terminar el proceso terapéutico.

Palabras Clave: adolescentes, psicoterapia, indicadores de cambios genéricos, adherencia
\end{abstract}

\begin{abstract}
This research is focused on the study Generic Change Indicators (GCI) in psychotherapies with adolescents conducted in natural settings. Specifically, it is centered on the evolution of GCI during the initial stage of therapy and their relation with treatment adherence. The participants were 19 adolescents with various diagnoses, who were receiving individual therapy with different theoretical approaches. Trained raters identified the CGI in the three initial sessions. The results reveal an evolution during the initial therapy stage, with hierarchically lower indicators dominating the first session and then decreasing in the second and third. In addition, the maximum CGI reached in the initial phase significantly predict the likelihood of completing the therapeutic process.

Keywords: adolescents, psychotherapy, generic change indicators, adherence
\end{abstract}

\footnotetext{
Correspondencia: Olga Fernández, ofernandezg@uc.cl

Este estudio recibió apoyo del Fondo de Innovación para la Competitividad (FIC) del Ministerio de Economía, Fomento y Turismo, a través de la Iniciativa Científica Milenio, Proyecto IS130005.
} 


\section{Introducción}

La adolescencia, con sus profundos cambios biopsicosociales, ha sido definida como una etapa de crisis (Erikson, 1968) y ha sido identificada como oportunidad de reparar funcionamientos o conflictos de etapas anteriores (Carvajal, 1994; Levisky, 1999; Quiroga, 2002). Desde esta perspectiva la psicoterapia en adolescentes se constituye en una herramienta privilegiada de intervención en un momento evolutivo de revisión y reacomodación de aspectos centrales para el desarrollo posterior, como lo es la identidad; en este mismo sentido, la psicoterapia en adolescentes puede constituir una acción preventiva para la salud mental de la futura población adulta (Kazdin, 2004; Russell, 2008).

La psicoterapia con adolescentes presenta ciertas características diferenciales en relación a la terapia con adultos, que la transforman en un objeto de estudio propio. Los adolescentes que asisten a tratamiento lo hacen en su mayoría por motivación de otros (padres, familia y/o escuela), ya que con frecuencia ellos no perciben la disfuncionalidad de su conducta (Kazdin, 2004). Además, en la misma terapia intervienen otros sujetos de forma directa o indirecta, como el sistema familiar, escolar o judicial, entre otros. A su vez, se ha planteado que características propias de la edad, como las necesidades de autonomía, autodeterminación, auto-confirmación y desconfianza de la autoridad adulta (Levisky, 1999; Russell, Shirk y Jungbluth, 2008) y el nivel de desarrollo cognitivo operatorio formal (Coll, Palacios y Marchesi, 2004), harían más complejo el establecimiento de una relación de ayuda. Todas estas características se han asociado a la dificultad en el establecimiento de la relación de ayuda en los adolescentes y su baja adherencia a los tratamientos.

Entender los procesos de cambio en la psicoterapia con adolescentes, específicamente conocer cómo operan los mecanismos básicos y condiciones esenciales para el inicio y desarrollo del proceso terapéutico, permitiría beneficiar a muchos adolescentes que -pese a que necesitan de ayudano permanecen en sus tratamientos. El presente estudio se focalizó en procesos de cambio en la fase inicial de la psicoterapia y su relación con la adherencia al tratamiento.

\section{Estudio del Proceso de Cambio: Fases, Momentos e Indicadores de Cambio Genéricos}

Las investigaciones en psicoterapia han identificado que el proceso terapéutico se desarrolla en diferentes fases que se presentan en sucesión jerárquica, donde la primera es requisito de la siguiente (Hill, 2005; Krause y Dagnino, 2005; Krause et al., 2007; Mergenthaler, 1996; Prochaska y DiClemente, 1984). Autores como Howard, Lueger, Maling y Martinovich (1993), Prochaska, DiClemente y Norcross, (1992), Hill (2005) y Krause (2005), han identificado que las etapas iniciales de la terapia son fundamentales para la instalación de la relación terapéutica, el desarrollo y resultados del proceso. Estas diversas conceptualizaciones del proceso de cambio coinciden en que el cambio supone avanzar por diferentes estados o fases y que las fases iniciales son de vital importancia para el establecimiento, desarrollo y resultado del proceso.

La investigación en procesos de cambio en psicoterapia también ha identificado eventos o momentos significativos que ocurren en las distintas fases de la terapia. Los segmentos significativos han recibido diferentes denominaciones, tales como Eventos de Empoderamiento (Timulak y Elliott, 2003), Momentos Innovativos (Gonçalves, Matos y Santos, 2009; Matos, Santos, Gonçalves y Martins, 2009), Insight (Elliott, 1984) y Momentos de Cambio (Krause et al., 2006; Reyes et al., 2008), entre otros.

Los Momentos de Cambio (MC), constructo teórico que se usará como marco conceptual de la investigación, son eventos o momentos significativos de la terapia donde se produce una transformación en los patrones subjetivos de interpretación y explicación (Krause, 2005; Krause et al., 2006). A través de la observación de los MC, Krause et al. (2006, 2007, 2014) han descrito un modelo de evolución del cambio psicoterapéutico genérico, es decir un modelo de cambio aplicable a procesos de distintas modalidades terapéuticas. Este modelo describe 19 Indicadores de Cambio Genéricos (ICG) (Figura 1). La evidencia indica que "es posible determinar el indicador a través de una verbalización de la o el paciente. Esta verbalización es indicativa del contenido del momento de cambio, es decir, permite ser asociada a un indicador de cambio específico, teniendo en consideración el contexto de la interacción terapéutica y del proceso de terapia" (Krause et al., 2006, p. 311). El ICG permite rotular el momento de cambio y da cuenta del nivel o estado en el proceso de cambio psicoterapéutico en que se encuentra el paciente.

Cada indicador de cambio tiene un número que lo identifica y ubica en un nivel dentro de la jerarquía de los cambios. Los 19 ICG pueden ser agrupados conceptualmente en tres estados de cambio (Altimir, Krause, de la Parra, Dagnino, Tomicic, Valdés, Echávarri, Vilches, Pérez, 2010), a saber; Nivel I: Consolidación inicial de la estructura de la relación terapéutica, el cual agrupa ICG del 1-7; Nivel II: Aumento 
1. Aceptación de la existencia de un problema.

2. Aceptación de los propios límites y reconocimiento de la necesidad de ayuda.

I. Consolidación inicial de la estructura de la relación terapéutica

II. Aumento de la permeabilidad hacia nuevos entendimientos

III. Construcción y consolidación de nuevos entendimientos
3. Aceptación del terapeuta como un profesional competente.

4. Expresión de esperanza.

5. Descongelamiento (o cuestionamiento) de formas habituales de entendimiento, comportamiento y/o emoción.

6. Expresión de la necesidad de cambio.

7. Reconocimiento de la propia participación en los problemas.
8. Descubrimiento de nuevos aspectos de sí mismo.

9. Manifestación de un comportamiento o emoción nuevos.

10. Aparición de sentimientos de competencia.

11. Establecimiento de nuevas asociaciones entre aspectos propios y del entorno, o entre aspectos propios y elementos biográficos.

12. Reconceptualización de los propios problemas y/o síntomas.

13. Transformación de valoraciones y emociones en relación a sí mismo u otros.

14. Formación de constructos subjetivos respecto de sí mismo a través de la interconexión de aspectos personales y del entorno, incluyendo problemas y síntomas.

15. Enraizamiento de los constructos subjetivos en la propia biografía.

16. Autonomía en cuanto al manejo del contexto de significado psicológico.

17. Reconocimiento de la ayuda recibida.

18. Disminución de la asimetría.

19. Construcción de una teoría subjetiva, biográficamente fundada, sobre sí mismo y la relación con el entorno.

Figura 1. Listado 19 Indicadores de Cambio Genérico

de la permeabilidad hacia nuevos entendimientos, que contiene los ICG del 8 al 13; y el Nivel III: Construcción y consolidación de nuevos entendimientos, el cual incluye los ICG del 14 al 19. Ciertos indicadores son observados más frecuentemente en etapas iniciales del proceso, mientras otros son característicos de etapas medias o finales de la psicoterapia (Krause 2005; Krause et al., 2006, 2007, 2015).

La investigación en ICG ha permitido establecer que los indicadores de cambio se presentan en un orden sucesivo, desde cambios iniciales hacia cambios más avanzados en la jerarquía, al avanzar en la terapia (Echávarri et al., 2009; Krause y Dagnino, 2005) y que se encuentran asociados positivamente a mejoría en el disconfort psicológico de los pacientes, aportando apoyo empírico a la noción de cambio subjetivo (Echávarri et al., 2009). También se ha observado que las terapias con mejores resultados finales muestran mayor presencia de ICG de Nivel III durante el proceso (Krause et al., 2015).

Sin embargo, los ICG surgen de la observación de psicoterapias con adultos y se han estudiado escasamente en psicoterapias con adolescentes, tanto en su forma de aparición como en relación con otras variables (por ejemplo la adherencia a la psicoterapia). Es así como el interés de este trabajo es relacionar los ICG de la primera fase del tratamiento con la permanencia en éste, teniendo como hipótesis que los cambios tempranos del o de la adolescente favorecerán su adherencia a la terapia.

\section{El problema de la Adherencia en Psicoterapia con Adolescentes}

Existen múltiples definiciones de adherencia y abandono (Deakin, Gastaud y Tiellet Nunes, 2012; Krebs, González, Rivera, Herrera y Melis, 2012), sin embargo, hay consenso sobre que el abandono sería la interrupción del tratamiento antes de alcanzar los objetivos terapéuticos propuestos (Gavino y Godoy, 1993); y que la adherencia correspondería a la permanencia en el tratamiento hasta alcanzar los objetivos terapéuticos. También se ha definido el abandono terapéutico como la terminación unilateral del tratamiento, siendo entonces la adherencia la continuación del tratamiento hasta una terminación acordada entre terapeuta y paciente (Pina, Silverman, Weems, Kurtines y Goldman, 2003).

La baja adherencia a la psicoterapia constituye un tema relevante a estudiar, dada su alta prevalencia y sus repercusiones sobre la efectividad de los tratamientos psicológicos en pacientes con problemas de salud mental (Issakidis y Andrews, 2004; Killaspy, Banerjee, King y Lloyd, 2000). Estudios poblacionales estiman que en contextos clínicos entre un 30 y un $50 \%$ de los ingresados para ser tratados o bien no comienzan el tratamiento o lo abandonan (Bados, Balaguer y Saldaña, 2007; Killaspy et al., 2000; 
Macharia, Leon, Rowe, Stephenson y Haynes, 1992). En el caso específico de la adherencia en pacientes adolescentes, como se mencionó anteriormente, las características propias del desarrollo dificultan el establecimiento de la relación de ayuda. Concordantemente, la evidencia empírica en psicoterapia con adolescentes ha registrado los problemas en el establecimiento de la relación y la adherencia al mismo, como una de las dificultades principales de las intervenciones en este grupo etario (Bronstein y Flanders, 1998; Kazdin, 2004; Manríquez, Molina y Zubarew, 2003; Martínez et al., 2009).

Si bien existe una gran cantidad de publicaciones relacionadas con la adherencia, la mayoría de ellas ha privilegiado la búsqueda de factores predictores del paciente, tales como variables sociodemográficas: nivel socioeconómico (Reis y Brown, 1999), edad y género (Bryant et al., 2007; Lambert, 2004); y variables clínicas: diagnóstico, severidad y cronicidad (Lambert, 2004; Porcel, 2005). También se han estudiado la alianza terapéutica (Mukherjee et al., 2006), los años de experiencia del terapeuta (Swift y Greenberg, 2012) y variables relativas al tratamiento: presencia o ausencia de coterapia farmacológica y el momento de su instalación (Edlund, Wang, Berglund, Katz, Lin y Kessler, 2002; Percudani, Belloni, Contini y Barbui, 2002). En contraste con esta abundancia de investigaciones, las variables relacionadas con el proceso y el micro-proceso se han estudiado escasamente en relación con la adherencia al tratamiento. Buscando aportar a llenar este vacío, la hipótesis específica de este estudio señala que los ICG de la etapa inicial en la psicoterapia con adolescentes se relacionarían positivamente con su adherencia al tratamiento.

\section{Método}

\section{Participantes}

Participaron 19 adolescentes asistentes a terapia individual en tres centros de atención ambulatorios de la ciudad de Santiago de Chile, de los cuales el $78.9 \%$ eran adolescentes mujeres y $21.1 \%$ hombres, con una edad promedio de 16.05 años $(D E=0.84$; rango 15 a 17 años). Los adolescentes fueron referidos a atención psicológica desde: Psiquiatría (57.9\%), Sistema Escolar (15.7\%), Familia (15.7\%) o consultaron en forma espontánea (10.5\%). Los diagnósticos consignados en sus fichas clínicas eran los siguientes: Trastorno Depresivo (8), Trastorno Ansioso (2),
Trastorno Conductual (3), Trastorno Adaptativo (3), Crisis de Desarrollo (2) y diagnóstico en estudio. (1).

Las terapias fueron de diferentes orientaciones teóricas: Sistémico / Constructivista (12), Psicodinámico (5) y Cognitivo-Conductual (2) y presentaron una duración promedio de 15.9 sesiones $(D E=10.67)$. Los terapeutas que desarrollaron las terapias fueron ocho (siete mujeres y un hombre), de una edad promedio de 34.6 años ( $D E=7.3$ ); con experiencia en el ejercicio de la psicoterapia de 9.4 años en promedio $(D E=4.3)$.

Al comparar aquellos adolescentes que finalizaron los procesos psicoterapéuticos respecto de aquellos que no lo hicieron, no se observaron diferencias significativas por sexo ( $p=.071$; estadístico exacto de Fischer) ni edad $(t$ : $-.31, g l, 17 p \leq .76$ ). Tampoco se observaron diferencias en los años de experiencia de los terapeutas entre las terapias que finalizaron y las que fueron interrumpidas $(t:-.29, g l$, $17 p \leq .077)$.

\section{Variables e Instrumentos}

Indicadores de Cambios Genéricos (ICG): Corresponden a la denominación que se le asigna a los Momentos de Cambios (MC) que ocurren dentro del proceso de cambio terapéutico, dando cuenta de contenidos del cambio, vale decir, de las sucesivas transformaciones en la representación de sí mismo del paciente (Krause et al., 2006, 2007, 2015).

Los MC pueden ser observados durante las sesiones terapéuticas, por evaluadores expertos entrenados (Krause, Arístegui y de la Parra, 2002), siguiendo los criterios de correspondencia teórica, verificabilidad, novedad y consistencia. La correspondencia teórica se refiere a que los contenidos del MC deben corresponder a alguno de los indicadores de cambio genéricos descritos (ver Figura 1). La verificabilidad se refiere a que el cambio debe ser observable (o mencionado) en la sesión. La novedad alude a que el fenómeno debe manifestarse por primera vez. Y, la consistencia "se refiere a la concordancia del cambio expresado verbalmente con las claves no-verbales, tanto generales como idiosincráticas de cada paciente y al hecho que los momentos posteriores de la sesión no deben contradecirlo" (Krause et al., 2006, p. 311).

El procedimiento general de codificación implica la observación de procesos psicoterapéuticos (en directo o video) por 2 o más jueces entrenados, quienes independientemente seleccionan los $\mathrm{MC}$ y los nominan conforme al listado de ICG, basados en el Manual de Observación y Codificación de los Episodios de Cambio y Estancamiento (Echávarri et al., 2009). Es importante mencionar que la identificación 
Tabla 1. Descripción de la muestra.

\begin{tabular}{|c|c|c|c|c|c|c|c|}
\hline Terapia & $\begin{array}{l}\text { Edad } \\
\text { Paciente }\end{array}$ & $\begin{array}{l}\text { Sexo } \\
\text { Paciente }\end{array}$ & Diagnóstico & $\begin{array}{l}\mathrm{N}^{\mathrm{o}} \\
\text { sesiones }\end{array}$ & Orientación teórica & $\begin{array}{l}\text { Años } \\
\text { Experiencia } \\
\text { Terapeuta }\end{array}$ & $\begin{array}{l}\text { Condición Finaliza } \\
\text { /Interrumpe }\end{array}$ \\
\hline I & 17 & $\mathrm{~F}$ & Crisis de Adolescencia & 7 & $\begin{array}{l}\text { Sistemica/ } \\
\text { Constructivista }\end{array}$ & 3 & Finaliza \\
\hline II & 17 & $\mathrm{~F}$ & Trastorno Conductual & 3 & $\begin{array}{l}\text { Sistemica/ } \\
\text { Constructivista }\end{array}$ & 9 & Interrumpe \\
\hline III & 15 & M & Trastorno Conductual & 3 & $\begin{array}{l}\text { Sistemica/ } \\
\text { Constructivista }\end{array}$ & 9 & Interrumpe \\
\hline IV & 15 & $\mathrm{~F}$ & Crisis de Adolescencia & 26 & $\begin{array}{l}\text { Sistemica/ } \\
\text { Constructivista }\end{array}$ & 9 & Finaliza \\
\hline V & 16 & $\mathrm{~F}$ & $\begin{array}{l}\text { Trstorno Depresivo } \\
\text { Moderado }\end{array}$ & 20 & Psicodinámica & 10 & Finaliza \\
\hline VI & 17 & $\mathrm{~F}$ & Trastorno de Ansiedad & 14 & $\begin{array}{l}\text { Sistemica/ } \\
\text { Constructivista }\end{array}$ & 8 & Finaliza \\
\hline VII & 17 & $\mathrm{~F}$ & $\begin{array}{l}\text { Trastorno de ansiedad } \\
\text { no/especificado }\end{array}$ & 30 & $\begin{array}{l}\text { Sistemica/ } \\
\text { Constructivista }\end{array}$ & 9 & Finaliza \\
\hline VIII & & & Informacion perdida & & & & \\
\hline IX & 16 & $\mathrm{~F}$ & Trastorno Depresivo severo & 8 & $\begin{array}{l}\text { Sistemica/ } \\
\text { Constructivista }\end{array}$ & 9 & Interrumpe \\
\hline $\mathrm{X}$ & 16 & $\mathrm{~F}$ & Trastorno Adaptativo & 34 & Psicodinámica & 10 & Finaliza \\
\hline $\mathrm{XI}$ & 16 & $\mathrm{~F}$ & Trastorno Depresivo severo & 10 & Psicodinámica & 10 & Interrumpe \\
\hline XII & 17 & $\mathrm{~F}$ & Trastorno Depresivo Severo & 16 & Psicodinámica & 10 & Interrumpe \\
\hline XIII & 15 & M & Trastorno Depresivo Severo & 16 & $\begin{array}{l}\text { Sistemica/ } \\
\text { Constructivista }\end{array}$ & 8 & Finaliza \\
\hline XIV & 16 & $\mathrm{~F}$ & Trastorno Adaptativo & 15 & Psicodinámica & 10 & Finaliza \\
\hline $\mathrm{XV}$ & 15 & $\mathrm{~F}$ & Trastorno Depresivo Severo & 31 & $\begin{array}{l}\text { Sistemica/ } \\
\text { Constructivista }\end{array}$ & 8 & Finaliza \\
\hline XVI & 15 & M & Trastorno Conductual & 12 & $\begin{array}{l}\text { Sistemica/ } \\
\text { Constructivista }\end{array}$ & 8 & Interrumpe \\
\hline XVII & 16 & M & $\begin{array}{l}\text { Trastorno depresivo } \\
\text { Moderado }\end{array}$ & 6 & Cognitivo-Conductual & 18 & Interrumpe \\
\hline XVIII & 17 & $\mathrm{~F}$ & Trastorno Depresivo severo & 34 & Cognitivo-Conductual & 18 & Finaliza \\
\hline XIX & 17 & M & Trastorno Adaptativo & 1 & $\begin{array}{l}\text { Sistemica/ } \\
\text { Constructivista }\end{array}$ & 12 & Interrumpe \\
\hline $\mathrm{XX}$ & 15 & $\mathrm{~F}$ & Diagnóstico en estudio & 16 & $\begin{array}{l}\text { Sistemica/ } \\
\text { Constructivista }\end{array}$ & 18 & Finaliza \\
\hline
\end{tabular}

de un MC, así como la especificación de su contenido conforme a los indicadores ICG es un proceso simultáneo, que se realiza a lo menos por dos evaluadores entrenados que observan y codifican las sesiones en forma independiente. Luego, después de un proceso de validación intersubjetiva, se seleccionaban los ICG en que ambos están de acuerdo. No se consideran los ICG en que no hay acuerdo. Una vez establecido el MC y su respectivo ICG, este es ratificado a través de la revisión de las transcripciones de las sesiones psicoterapéuticas.

Adherencia: Para efectos de este estudio fue definida como la continuación del tratamiento hasta una terminación acordada entre terapeuta y paciente (Pina et al., 2003). Esta condición se registró como una variable dicotomizada, donde la condición "finaliza" da cuenta de que el/la adolescente termina el proceso en acuerdo con el terapeuta, en cambio, la condición "interrumpe" indica que el paciente deja de asistir a terapia sin conversarlo y/o acordarlo con el terapeuta.

\section{Procedimiento}

Se contactaron instituciones de la ciudad de Santiago de Chile que brindan atención psicológica individual, de distintas modalidades teóricas, a adolescentes y que tuvieran convenio de pago con los distintos prestadores de servicios de salud de la población, de modo que las terapias estudiadas se realizaron en contexto natural. Las instituciones fueron seleccionadas en forma intencional y 
por criterio de accesibilidad. Los terapeutas, pacientes y sus padres dieron su asentimiento y consentimiento por escrito para grabar en video las tres sesiones iniciales y completar los cuestionarios. El estudio contó con la aprobación del Comité de Ética de la Escuela de Psicología de la Pontificia Universidad Católica de Chile.

La identificación de los ICG, como se señaló anteriormente, fue realizada por dos evaluadores expertos, quienes en forma independiente observaron los videos y leyeron las transcripciones de las tres sesiones iniciales, consignando el número y tipo del indicador, el turno de palabra y el momento de la sesión donde se identificó. Posteriormente, se llevó a cabo un proceso de consenso intersubjetivo entre los jueces y sólo se consideraron para la investigación los ICG donde hubo acuerdo entre los evaluadores. Para los análisis se consideró el número del ICG (identificación), su nivel en la jerarquía de cambios (Nivel I, II y III) y el ICG máximo alcanzado en cada una de las tres primeras sesiones para cada terapia.

\section{Análisis de Datos}

En primera instancia se realizó el análisis descriptivo del comportamiento de los ICG en las tres sesiones iniciales. Luego se realizó un análisis de frecuencia por sesión y un análisis de frecuencia por niveles de cambio (Nivel I, Nivel II y Nivel III), a modo de describir el patrón de presentación, la evolución y trayectoria de los ICG en la fase inicial (diferencias de frecuencia $\mathrm{Chi}^{2}$, Anova de mediciones repetidas).

Para la relación entre ICG y Adherencia se realizó Anova simple entre promedios de ICG máximo alcanzado en terapias que finalizan /interrumpen y Regresión Logística Bivariada entre ICG y adherencia dicotomizado.

\section{Resultados}

\section{Evolución de los ICG}

En la Tabla 2 se muestran las frecuencias y porcentajes de presentación de los ICG según su nivel jerárquico en cada una de las 3 primeras sesiones de la psicoterapia.

Nota. $* p \leq .05 * * p \leq .01 * * * p \leq .001$ Se observa que en la sesión uno es más frecuente, que lo esperado por el azar, encontrar ICG del Nivel I que del Nivel II, en cambio en la sesión 3 es más frecuente encontrar ICG del Nivel II que del Nivel I $(\chi 2(2, \mathrm{~N}=66)=18.14,, p \leq .001)$. A su vez, al comparar la probabilidad de ocurrencia de ICG de Nivel I y II entre las sesiones dos y tres se encontraron diferencias
Tabla 2. Distribución de los Indicadores de Cambio Genérico por sesión y por Niveles de Cambio.

\begin{tabular}{lcccccc}
\hline & \multicolumn{2}{c}{ Sesion 1 } & \multicolumn{2}{c}{ Sesión 2 } & \multicolumn{2}{c}{ Sesión 3 } \\
& F & $\%$ & F & $\%$ & F & $\%$ \\
\hline ICG Nivel I & $35^{* *}$ & 89.7 & 17 & 71 & 11 & 40.7 \\
ICG Nivel II & 4 & 10.3 & 7 & 29 & $16^{*}$ & 59.3 \\
ICG Nivel III & 0 & 0 & 0 & 0 & 0 & 0 \\
Total & 39 & 100 & 24 & 100 & 27 & 100 \\
\hline
\end{tabular}

Tabla 3. Promedio y desviación estándar de Indicadores de Cambio Genéricos máximos por sesión

\begin{tabular}{lccc}
\hline & $\mathrm{N}$ & Media & Desv.Típ. \\
\hline ICG_max Sesión 1 & 19 & 3.632 & 3.578 \\
ICG_max Sesión 2 & 14 & 5.071 & 4.341 \\
ICG_max Sesión 3 & 14 & $7.357^{* *}$ & 4.012 \\
\hline
\end{tabular}

Nota. $* p \leq .05 * * p \leq .01 * * * p \leq .001$

significativas $(\chi 2(2, \mathrm{~N}=51)=4.65, p \leq .032)$, aumentando la probabilidad de aparición de cambios Nivel I en las segundas sesiones y de cambios Nivel II en las terceras sesiones. Al comparar las sesiones uno versus dos, en cuanto a la probabilidad de ocurrencia de ICG de Nivel I vs Nivel II, no se observan probabilidades diferentes al azar $(p \leq$ .0863 , estadístico exacto de Fischer).

Otra forma de aproximarse a observar la evolución de los ICG fue considerar el ICG más alto alcanzado en cada una de las tres primeras sesiones (Tabla 3). Existe una diferencia significativa entre los promedios de los ICG de la primera respecto de la tercera sesión $(F(1,13=10.843, p=$ .006). Coherente con los resultados antes presentados, no se observan diferencias significativas al comparar los promedio de los ICG de primera y segunda sesión $(F(1,13)=1.103$, $p=.313)$, ni entre el promedio de los ICG de la segunda y tercera sesión $(F(1,10)=2.668 p=.133)$.

\section{Cambio en la etapa inicial de la psicoterapia y adherencia}

Para responder la pregunta sobre la relación del nivel jerárquico de los ICG iniciales y la adherencia a la psicoterapia, se realizó un modelo de regresión logística binaria para determinar la probabilidad de finalizar la terapia $(1=$ finaliza psicoterapia y $0=$ interrumpe), utilizando como predictor el ICG máximo alcanzado en cada una de las psicoterapias en la fase inicial. Como se observa en la Tabla 3, el modelo indica que la probabilidad de terminar la terapia es predecible por el ICG máximo alcanzado en 
la fase inicial de la terapia. Así, en la medida que aumenta en un punto (pasa de un ICG al siguiente) la probabilidad de finalizar psicoterapia aumenta 1.45 veces. Por ejemplo, este modelo especifica que hay un $32.59 \%$ de finalizar la psicoterapia cuando el ICG máximo en las primeras sesiones es 4 , aumentando a $41.14 \%$ la probabilidad de finalizar la psicoterapia cuando el ICG máximo es 5.

\section{Discusión}

Los Indicadores de Cambio Genéricos (ICG) en la fase inicial de la psicoterapia con adolescentes presentaron una evolución desde una primacía de cambios relacionados con la Consolidación Inicial de la Estructura de la Relación Terapéutica (Nivel I) hacia cambios relacionados con el Aumento de la Permeabilidad hacia Nuevos Entendimientos (Nivel II), observándose una diferencia significativa en la presencia de ICG de Nivel I y Nivel II entre la sesión uno y la tres. Este resultado aporta evidencia empírica sobre la evolución al interior de la etapa inicial de la terapia, en el sentido de un predominio de los indicadores jerárquicamente más bajos en la primera sesión en relación a la segunda y la tercera. Este patrón de presentación de los ICG observado confirma la evolución del cambio psicoterapéutico encontrada en terapias con adultos (Krause et al., 2007, 2015) y es coherente con los postulados teóricos del modelo (Krause, 2005, 2011).

Los resultados expuestos también muestran la relación positiva de los ICG con la adherencia, en los dos análisis realizados. Por una parte, el promedio de los ICG máximos alcanzados en la fase inicial es significativamente más alto en las terapias que finalizan y por otra, el ICG máximo alcanzado en la terapia permite predecir en forma significativa la probabilidad de terminar el proceso terapéutico. Este resultado da cuenta que los cambios tempranos son cruciales para que el paciente adolescente se mantenga en terapia. Posiblemente el mayor cambio subjetivo, reflejado en las ICG de más alto nivel jerárquico, se vincula con una mayor involucramiento motivación del paciente con el proceso terapéutico, facilitando así su permanencia en él.

Desde el punto de vista de la práctica clínica, es beneficioso poder reconocer en qué estadio de cambio se encuentra el paciente, específicamente en el caso de los adolescentes, donde se ha descrito escasa motivación por consultar (Kazdin, 2004). Si el trabajo en sesión se ajusta al nivel de representación del problema que trae el joven, sería más probable que le haga sentido el trabajo terapéutico, que se motive y que permanezca en terapia, de modo de
Tabla 4. Parámetros del modelo de regresión logística que predice finalizar $v / s$ interrumpir terapia según ICG máximo alcanzado.

\begin{tabular}{lllllll}
\hline & B & E.T. & Wald & gl & Sig. & $\operatorname{Exp}(\mathrm{B})$ \\
\hline Predictores & & & & & & \\
ICG máximo & $0.369^{*}$ & $0.158^{*}$ & 5.43 & 1 & .02 & 1,45 \\
Constante & -2.203 & 1.214 & 3.291 & 1 & .07 & 0,11 \\
\hline
\end{tabular}

Nota. ${ }^{*} p \leq .05 * * p \leq .01 * * * p \leq .001$

dar ocasión a seguir avanzando en su proceso de cambio. Los cambios iniciales dentro de la jerarquía de ICG dicen relación con reconocer la existencia de un problema, aceptar los propios límites y la necesidad de ayuda, aceptar al terapeuta como profesional competente, entre otros. Entonces, en las primeras sesiones es esencial identificar la existencia de un problema y estar dispuesto a trabajar en su solución con un otro -el terapeuta- definido como experto. Si esto no se ha logrado, es necesario trabajar en ello, antes de seguir revisando otros aspectos, porque de lo contrario es probable que el joven desista de asistir. De este modo, los ICG no sólo se transforman en un instrumento útil para la investigación del proceso de cambio psicológico, sino que se presentan como una herramienta de utilidad clínica, posibilitando establecer los deseados puentes entre la clínica y la investigación (Teachman, Drabick, Hershenberg, Vivian, Wolfe y Goldfried , 2012).

En cuanto a las limitaciones del estudio, el tamaño de la muestra no permitió realizar análisis más específicos por patología de los adolescentes, modalidades terapéuticas y /o años de experiencia del terapeuta, constituyendo el análisis del comportamiento de los ICG en función de esas variables un desafío para futuros estudios. También sería relevante comparar los ICG, --como indicadores de cambio subjetivo generados a través de un dispositivo observacional- con la evaluación de los logros alcanzados en la terapia desde las perspectivas de pacientes y terapeutas, a través de entrevistas de seguimiento que permitan explorar la vivencia subjetiva de los cambios.

\section{Referencias}

Altimir, C., Krause, M., de la Parra, G., Dagnino, P., Tomicic, A., Valdés, N., Echávarri, O., Vilches, O., y Pérez, J. C., (2010). Clients', therapists and observers> perspectives on moments and contents of therapeutic change. Psychotherapy Research, 20, $472-487$.

Bados, A., Balaguer, G., y Saldaña, C. (2007). The efficacy of cognitivebehavioral therapy and the problem of drop-out. Journal of Clinical Psychology, 63, 585-592. doi:10.1002/jclp.20368

Bronstein, C., y Flanders, S. (1998). The development of a therapeutic space in first contact with adolescents. Journal of child psychotherapy, 24, 5-35.doi.org/10.1080/00754179808414803 
Bryant, R. A., Moulds, M. L., Mastrodomenico, J., Hopwood, S., Felmingham, K., y Nixon, R. D. V. (2007). Who drops out of treatment for post-traumatic stress disorder? Clinical Psychologist, 11, 13-15. doi:10.1080/13284200601178128

Carvajal, G. (1994). Adolecer: La Aventura de una metamofosis. Una visión psicoanalítica de la adolescencia. Santafé de Bogotá: Tiresias.

Coll, C., Palacios, J., y Marchesi, A. (2004). Desarrollo Psicológico y Educación. Madrid: Alianza.

De la Parra, G., von Bergen, A. y Del Río, M. (2002). Primeros Hallazgos de la Aplicación de un instrumento que mide resultados Psicoterapéuticos en una muestra de pacientes y de población general. Revista Chilena de Neuropsiquiatría, 40, 201-209.

Deakin, E., Gastaud, M., y Tiellet Nunes, M. L (2012) Child psychotherapy dropout: an empirical research review. Journal of Child Psychotherapy, 38, 2, 199-209.

Echávarri, O., González, A., Krause, M., Tomicic, A., Pérez, C., Dagnino, P., de la Parra, G., Valdés, N., Altimir, C., Vilches, O., Strasser, K., Ramírez, I., y Reyes, L. (2009). Cuatro terapias psicodinámicas breves exitosas estudiadas a través de los indicadores genéricos de cambio. Revista Argentina de Clínica Psicológica, 18, 5-19.

Edlund, M. J., Wang, P. S., Berglund, P. A., Katz, S. J., Lin, E., y Kessler, R. C. (2002). Dropping out of mental health treatment: Patterns and predictors among epidemiological survey respondents in the United States and Ontario. The American Journal of Psychiatry, 159, 845-851. doi:10.1176/appi.ajp.159.5.845.

Elliott, R. (1984). A discovery-oriented approach to significant change events in psychotherapy: Interpersonal process recall and comprehensive process analysis. In L. Rice y L. S. Greenberg (Eds.), Patterns of change: Intensive analysis of psychotherapy process (pp. 249-286). New York: Guilford Press.

Erikson, E. (1968). Identidad, juventud y crisis. Buenos Aires, Paidós.

Gavino, A., y Godoy, A. (1993). Motivos de abandono en terapia de conducta. Análisis y Modificación de Conducta, 19, 511-536.

Gonçalves, M.M., Matos, M., y Santos, A. (2009). Narrative therapy and the nature of "innovative moments" in the construction of change. Journal of Constructivist Psychology, 22, 1-23

Hill, C. (2005). Therapist Techniques, Client Involvement, and the therapeutic Relationship: inextricably intertwined in the Therapy Process. Psychotherapy: Theory, Research, Practice, Training, 42, 431-442.

Howard, K. L., Lueger, R. J., Maling, M. S., y Martinovich, Z. (1993). A phase model of psychotherapy outcome: causal mediation of change. Journal of Consulting and Clinical Psychology, 61, 678-85

Issakidis, C., y Andrews, G. (2004). Pretreatment attrition and dropout in an outpatient clinic for anxiety disorders. Acta Psychiatrica Scandinavica, 109, 426-433. doi:10.1111/j.1600-0047.2004.00264.x.

Kazdin, A. (1990). Premature termination from treatment among children referred for antisocial behavior. Journal of Child Psychology and psychiatry and Allied Disciplines, 31, 415-425.

Kazdin, A (2004). Psychotherapy for Children and Adolescents. In M. Lambert (Ed.), Bergin and Garfield's Handbook of psychotherapy and behavior change. New York: Wiley and Sons.

Killaspy, H., Banerjee, S., King, M., y Lloyd, M. (2000). Prospective controlled study of psychiatric out-patient non-attendance: Characteristics and outcome. The British Journal of Psychiatry, 176, 160-165. doi:10.1192/bjp.176.2.160.

Krause, M. (2005). Psicoterapia y Cambio. Una mirada desde la subjetividad. Ediciones Universidad Católica de Chile, Santiago Chile.

Krause, M., Altimir, C., y Horvath, A. (2011). Deconstructing the Therapeutic Alliance: Reflections on the Underlying Dimensions of the Concept. Clínica y Salud, 22, 267-283.

Krause, M., Aristegui, R., De la Parra, G., y Strasser, K. (2006). Facilitando el cambio en psicoterapia: interacciones comunicacionales efectivas e inefectivas en diferentes modalidades psicoterapéuticas. (2006-2007) Proyecto financiado por FONDECYT Nº 1060768

Krause, M., De la Parra, G., Arístegui, R., Tomicic, A., Dagnino, P., Echávarri, O., Valdés, N. Vilches, O., Ben-Dov, P., y Altimir, C. (2006). Indicadores de cambio genéricos en la investigación psicoterapéutica, Revista Latinoamericana de Psicología, 38, 299-325.
Krause, M., y Dagnino, P. (2005/2011). Evolución del cambio en el proceso terapéutico. En: E. Thumala y R. Riquelme, (eds), Avances en Psicoterapia: Cambio Psíquico y Vínculo (pp. 21-36). Santiago de Chile: Sociedad Chilena de Salud Mental.

Krause, M., De la Parra, G., Aristegui, R., Dagnino, P., Tomicic, A., Valdés, N., Echávarri, O., Strasser, K., Reyes, L., Altimir, C., Ramirez, I., Vilches, y O Ben-dov, P. (2007). The evolution of therapeutic change studied through generic change indicators. Psychotherapy Research, 17, 673-689.

Krause, M., Pérez, J. C., Altimir, C., y de la Parra, G. (2015). Generic Change Indicators in Therapeutic Processes with Different Outcomes. Psychotherapy Research, 25, 533-545. doi: 10.1080/10503307.2014.935516.

Krebs, M., González, L. M., Rivera, A., Herrera, P., y Melis, F. (2012) Adherencia a Psicoterapia en Pacientes con Trastornos de Ansiedad. Psykhe, 21, 133-147.

Lambert, M. (2004). Bergin and Garfield's handbook of psychotherapy and behavior change ( $5^{\mathrm{a}}$ ed.). New York, NY: John Wiley y Sons.

Levisky, D. (1999). Adolescencia: reflexiones psicoanaliticas. Buenos Aires: Lumen.

Macharia, W. M., Leon, G., Rowe, B. H., Stephenson, B. J., y Haynes, R. B. (1992). An overview of interventions to improve compliance with appointment keeping for medical services. JAMA: The Journal of the American Medical Association, 267, 1813-1817. doi:10.1001/ jama.1992.03480130129038.

Manríquez, H., Molina, H. y Zubarew, T. (2003). Promoción y prevención de salud en la adolescencia. En T. Zubarew, M. Romero, y F. Poblete (Eds.), Adolescencia, promoción, prevención y atención de salud. Santiago: Ediciones Universidad Católica de Chile.

Martínez, V., Martínez, P., Poblete, C., Chang, M., Brand, L., y Soto, P (2009). Psicoterapia para Adolescentes en programa GES de depresión de un centro privado de Salud Mental. Revista Chilena de psiquiatría y Neurología de la Infancia y Adolescencia, 19,71-72.

Matos, M., Santos, A., Gonçalves, M., y Martins, C. (2009). Innovative moments and change in narrative therapy. Psychotherapy Research, $19,68-80$

Mergenthaler, E (1996). Emotio-Abstraction Patterns in verbatim protocols: anew way of describing psychotherapeutic processes. Journal of Counsulting and Clinical Psychology, 64, 1306-1318.

Mukherjee, S., Sullivan, G., Perry, D., Verdugo, B., Means-Christensen, A., Schraufnagel, T., y Roy-Byrne, P. P. (2006). Adherence to treatment among economically disadvantaged patients with panic disorder. Psychiatric Services, 57, 1745-1750. doi:10.1176/appi.ps.57.12.1745.

Percudani, M., Belloni, G., Contini, A., y Barbui, C. (2002). Monitoring community psychiatric services in Italy:Differences between patients who leave care and those who stay in treatment. The British Journal of Psychiatry, 180, 254-259. doi:10.1192/bjp.180.3.254

Pina, A. A., Silverman, W. K., Weems, C. F., Kurtines, W. M., y Goldman, M. L. (2003). A comparison of completers and noncompleters of exposure-based cognitive and behavioral treatment for phobic and anxiety disorders in youth. Journal of Consulting and Clinical Psychology, 71, 701-705. doi:10.1037/0022-006X.71.4.701.

Porcel, M. (2005). El abandono en las terapias psicológicas. Aposta: Revista de Ciencias Sociales, 14, 1-16.

Prochaska, J., DiClemente, C., y Norcross, J. (1992). In search of how people change. American Psychologist, 47, 1102-1114.

Prochaska, J. O., y DiClemente, C.C. (1984). The transtheoretical approach: Crossing the traditional boundaries of therapy. Melbourne, Florida: Krieger Publishing Company. ISBN: 13: 978-0894648489.

Quiroga, S (2002). Adolescencia: del goce orgánico al hallazgo del objeto. Buenos Aires: Eudeba.

Reis, B. F., y Brown, L. G. (1999). Reducing psychotherapy dropouts: Maximizing perspective convergence in the psychotherapy dyad. Psychotherapy, 36, 123-136. doi:10.1037/h0087822.

Reyes, L., Arístegui, R., Krause, M., Strasser, K., de la Parra, G., Tomicic, A., Dagnino, P., Vilches, O., Valdés, N., Echávarri, O., Altimir, C., Ramírez, I., y Ben-Dov, P. (2008). Language and Therapeutic Change: A Speech Acts Analysis. Psychotherapy Research, 18, 355-362. 
Russell, R. (2008). Child and Adolescent psychotherapy: Introduction to the special section. Psychotherapy Research, 18, 1-4.

Russell, R., Shirk, S., y Jungbluth, N. (2008). First-session pathways to the working alliance in cognitive behavioral therapy for adolescent depression. Psychotherapy Research, 18, 15- 27.

Swift, J. K., y Greenberg, R. P. (2012). Premature discontinuation in adult psychotherapy: A meta-analysis. Journal of Consulting and Clinical Psychology, 80, 547-559. doi:10.1037/a0028226.

Timulak, L., y Elliott, R. (2003). Empowerment Events in ProcessExperiential Psychotherapy of Depression: An exploratory qualitative analysis. Psychotherapy Research, 13, 443-460.

Teachman, B. A., Drabick, D. A., Hershenberg, R., Vivian, D., Wolfe, B. E., y Goldfried, M. R. (2012). Bridging the gap between clinical research and clinical practice: introduction to the special section. Psychotherapy, 49, 97-100. doi: 10.1037/a0027346. 
